\title{
Smart Volume Controller for Mobile Phones
}

\author{
Laxman Singh, Sunil Kumar Chaudhary, Yogesh Kumar Verma, Jay Kant Pratap Singh Yadav, Rajeev \\ Kumar
}

\begin{abstract}
In this paper, smart volume controller (SVC) using fuzzy logic is developed for mobile phones in order to improve the voice quality in the presence of background noise. The SVC uses the noise level and class information as an input to automatically raise the volume of the cell phone in the presence of background noise. Smart volume controller mainly consists of two stages: (i) Noise Classification, (ii) Fuzzy Volume Controller. Noise classification includes feature extraction and feature matching using artificial neural network classifier to differentiate between different types of noise classes. The maximum noise attenuation level of up to $55 \mathrm{db}$ was achieved while experimenting with the four different types of noises such as car noise, market noise, office noise, and train noise using the proposed volume controller that seems to be quite satisfactory.
\end{abstract}

Keywords: Smart volume controller, Mel frequency Cepstral coefficients, linear predictive coefficients, Real cepstral parameter coefficients.

\section{INTRODUCTION}

Whenever, a conversation is carried out on a mobile phone in the presence of high background noise level, speaker on the other end is often asked to speak up or to come out of a noisy place. Otherwise, user needs to bring their mobiles very near to their ears in the presence of high level of noise. Therefore, in this paper, we have made an attempt to improve the quality of service by developing a smart volume controller that could automatically raise the volume of mobile phones. The noise level derived from the voice activity detector (VAD) present within the speech codec, the current volume level, and the noise class acts as an input to the fuzzy volume controller.

Background noise levels may be high while travelling in busses, trains, planes or while passing through markets, sporting venues, and other public places. Background noise classification information is very useful and can be used to dynamically follow the changes in acoustic volume levels in different noise environments. The fuzzy volume controller for mobile phones will have the capability to adjust the volume according to the background noise level and noise class using a fuzzy expert system. By intelligently adjusting

Revised Manuscript Received on December 30, 2019.

* Correspondence Author

Laxman Singh*, Dept. of Elect. \& Comm. Engineering, Noida Institute of Engineering \& Technology, Gr. Noida, India.

Sunil Kumar Chaudhary, Dept. of EE,Galgotias College of Engg. \& Tech., (Affiliated with Dr. A.P.J.Abdul Kalam Tech. University), U.P, India, Yogesh Kumar Verma, Dept. of Elect. \& Comm. Engineering, Lovely Professional University, Phagwara, Punjab, India

Jay Kant Pratap Singh Yadav, Dept. of Computer Science \& Engg., Ajay Kumar Garg Engineering College, Ghaziabad, U.P., India

Rajeev Kumar, Department of Master of Computer Applications, Noida Institute of Engineering \& Technology, Gr. Noida, India.

(C) The Authors. Published by Blue Eyes Intelligence Engineering and Sciences Publication (BEIESP). This is an open access article under the CC BY-NC-ND license (http://creativecommons.org/licenses/by-nc-nd/4.0/) the volume level, the Quality of service can be improved for both stationary and non-stationary background noise in mobile environments. Hearing loss in individuals can be gradual, and quality of hearing can vary from person to person. Hearing loss can also result in difficulty for individuals in understanding speech in the presence of background noise. Hence, the SVC needs to be personalized based on the requirements of an individual's hearing requirements of understanding speech in the presence of background noise.

\section{MATERIAL AND METHODS}

In this experiment, all the audio samples used for this study were collected from different noisy places with the help of microphone attached to the Laptop. Fig.1 shows the implementation process of the fuzzy based smart volume controller. The implementation process consisted of data acquisition and preparation, Noise classifier model construction followed by the fuzzy volume controller model.

\section{A. Data Acquisition}

In our research work, four different types of audio samples such as car, train, market place, and office were collected from different places of Northern Capital Region (NCR), India using microphone attached to the Laptop and were processed in MATLAB 2018a environment. Two hundred samples of each type of noise totalling to 800 noise samples were used to develop the proposed model.

\section{B. SMART VOLUME CONTROLLER}

The manual volume control has several shortcomings. For a sophisticated mobile phone, the user needs to be trained to use volume control to get the better sound quality. A volume controller that has the capability to intelligently adjust the volume levels is referred as Smart Volume Controller (SVC) [1]. Design and development of the SVCs depends upon the very complex models of hearing mechanism as well as the measurement of inputs required for SVC such as background noise level, loudness level, and noise class.

Noise classes like car noise fall into low-frequency noise. They do not affect the intelligibility of speech so much as factory noise. Therefore, the use of background noise and class information can provide effective volume adjustment. Depending upon the characteristics of the data; the quest for a suitable model is a pertinent challenge. In this work, an smart volume controller is designed and its performance is observed in terms of noise attenuation level.

Best noise parameter estimate for smart volume controller is done using artificial neural network (ANN) [2] in order to get accurate noise classes to produce the better results. The block diagram of the SVC is as shown in Fig.1.

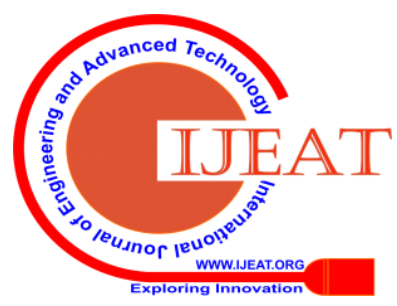




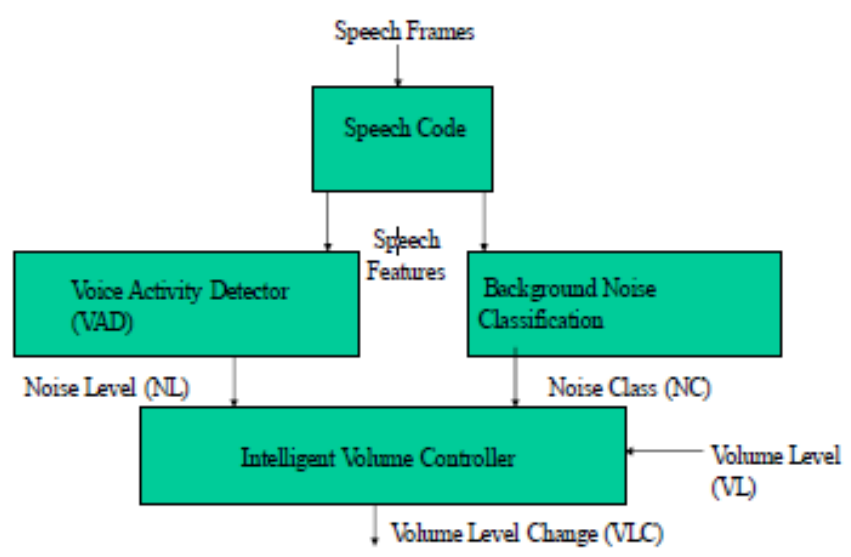

Fig. 1. Block diagram of Smart Volume Controller

The SVC model consists of the following module-
(a) Noise feature extraction
(b) Noise level detector (NLD)
(c) Background Noise Classifier (BNC)
(d)Fuzzy Volume Controller(FVC)

These modules are discussed in the subsequent sub-sections as follows:

\section{(a) Noise Feature Extraction}

In this block, framing was carried out of all the different types of noise samples and noise features such as Mel Frequency Cepstral coefficient (MFCC), Real Cepstral parameter (RCEP), and Linear predictive coding (LPC) were computed to analysis the noise through any one of the soft computing techniques viz., artificial neural networks (ANN), fuzzy logic, genetic algorithms or a combination of these. However, in our experiment, we used ANN for this purpose due to its easy implantation and good performance for the complex noisy signals [4].

MFCC: In order to compute the set of MFCC, noise frame of around 30msec length was used for each sample. MFCC is considered as a powerful technique to extract features from the noisy signals [3]. The MFCC feature vector is computed by integration of the spectrum within triangular bins arranged on a Mel frequency axis that generates a mel frequency binned spectrum and is further processed by a log and DCT operations. The MFCC vectors and pitch conserve sufficient information for continuous speech recognition (at various rates of error). Despite of that, it is not necessary to regenerate good quality speech from them due to having lost a significant amount of information during the feature extraction.

RCEP: Theoretically, the real Cepstrum represents the inverse Fourier transform of the real logarithm of the magnitude of Fourier transform. For getting smooth harmonic structure of the spectrum, first few cepstral coefficients are retained only setting other to be zero. Cepstral coefficients are very useful coefficients for representing the noise spectral envelope.

This can be expressed as :

$$
y=\frac{1}{2 \pi} \int_{-\pi}^{\pi} \log \left|X\left(e^{i w t}\right)\right| e^{i w t} d w
$$

Where $\mathrm{x}$ represent the signal whose spectrum is to be computed. The above equation computes the Fourier Transform of $\mathrm{x}$, therefore, can be employed for the separation of two signals convolved with each other. Therefore, RCEP is considered an efficient technique mainly used for computing the Harmonics-to-Noise Ratio (HNR) in Noise Signals.

LPC: The logic behind considering the linear prediction as an important feature is that a noise sample can be expressed as a linear combination of past noise samples. In order to get the unique set of predictor coefficients, we need to minimize the sum of the squared differences between the actual noise samples and the predicted ones. The LPC algorithm is known as a conventional method that could be utilized to predict the forthcoming sample from the previous samples considering the mean squared error to be minimum [5].

\section{(b) Noise level detector (NLD)}

The noise level detector produces noise level (NL) in decibel (DB) as an output. The artificial neural network (ANN) acts here as a noise level detector whose output is fed to Smart Volume Controller as one of the input features.

\section{(c) Background Noise Classifier (BNC)}

Noise features such as MFCC, RCEP, and LPC are used as an input to the ANN classifier to yield the noise class. The noise class is further used as an input to Fuzzy expert system to control the volume level as per the noise class and noise level. Thus VLC (volume level change) was observed maximum in case of train and minimum in case of office.

\section{(d) Fuzzy Volume Controller (FVC)}

The proposed fuzzy volume controller (FVC) [6-8] was developed using the fuzzy logic toolbox available in MATLAB 2018a. The above three models of FVC model give volume level change (VLC) based on inputs viz., noise level, noise class, and volume level of original speech samples. Based on these inputs, volume level of mobile phone was varied in presence of background noise and its level was raised accordingly.

\section{INTELLIGENT VOLUME CONTROLLER}

In this section, the Fuzzy expert system is used to design an intelligent volume controller (IVC). To design, we used the triangular membership function and using fuzzy 40 if-then fuzzy rules. Volume level (VL) was analyzed for four categories of internet noises from $0.1 \mathrm{~dB}$ to $100 \mathrm{~dB}$ and it was also normalized between 0 to 1 for defining the membership function in fuzzy for : (a) Office Noise, (b) Market Noise, (c) Car Noise, and (d)Train Noise

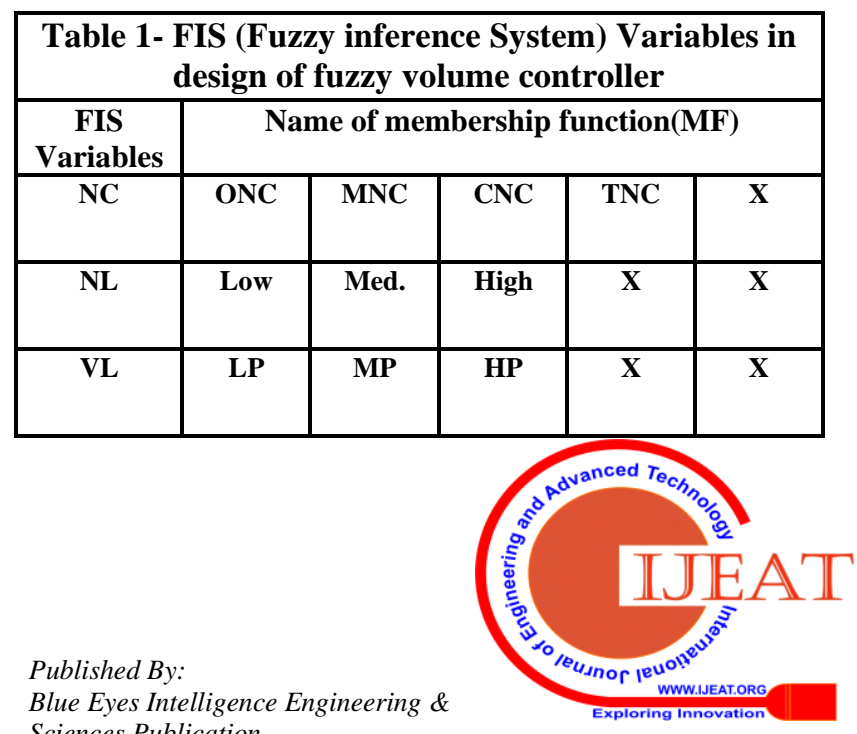



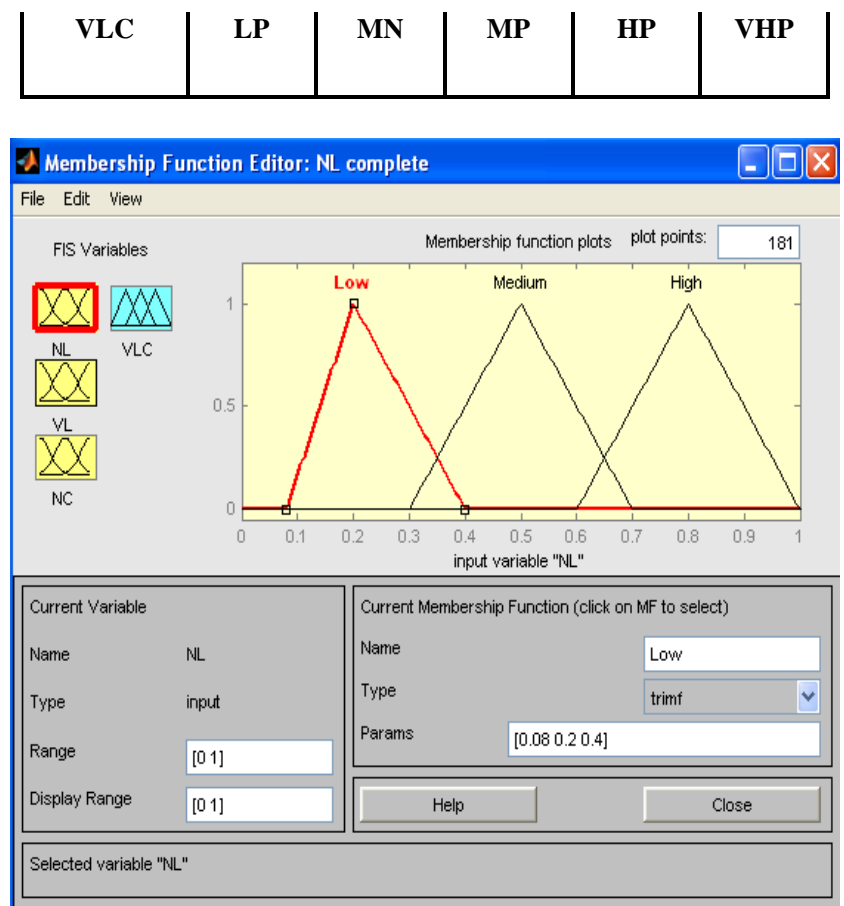

Fig.2. Noise level defined by the triangular Fuzzy Membership functions.

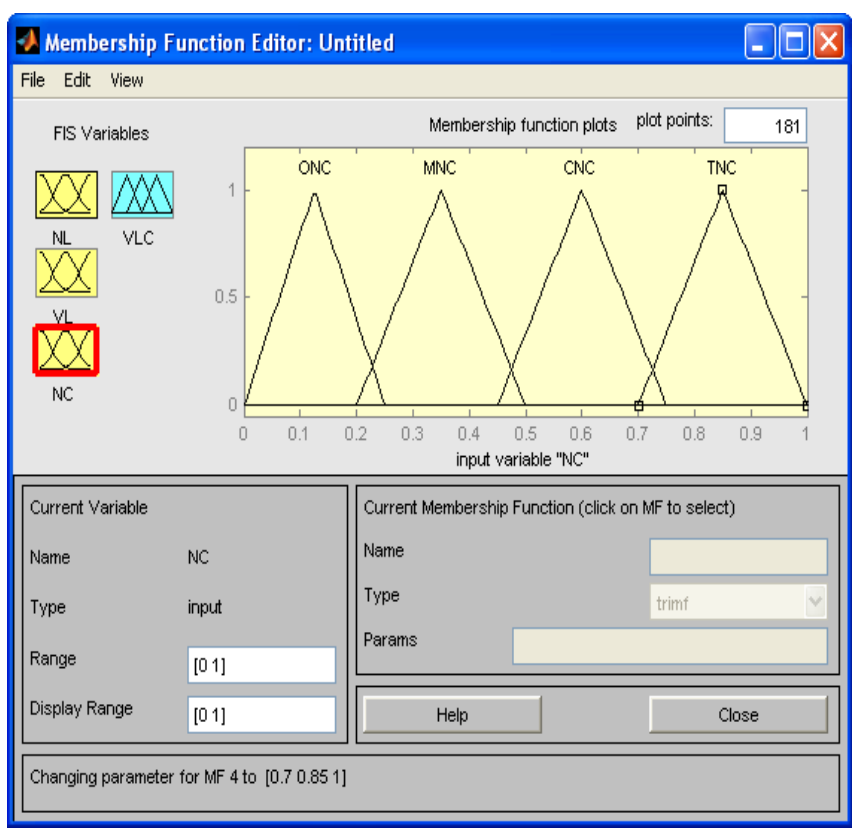

Fig. 3. Noise Class defined by the triangular Fuzzy Membership functions

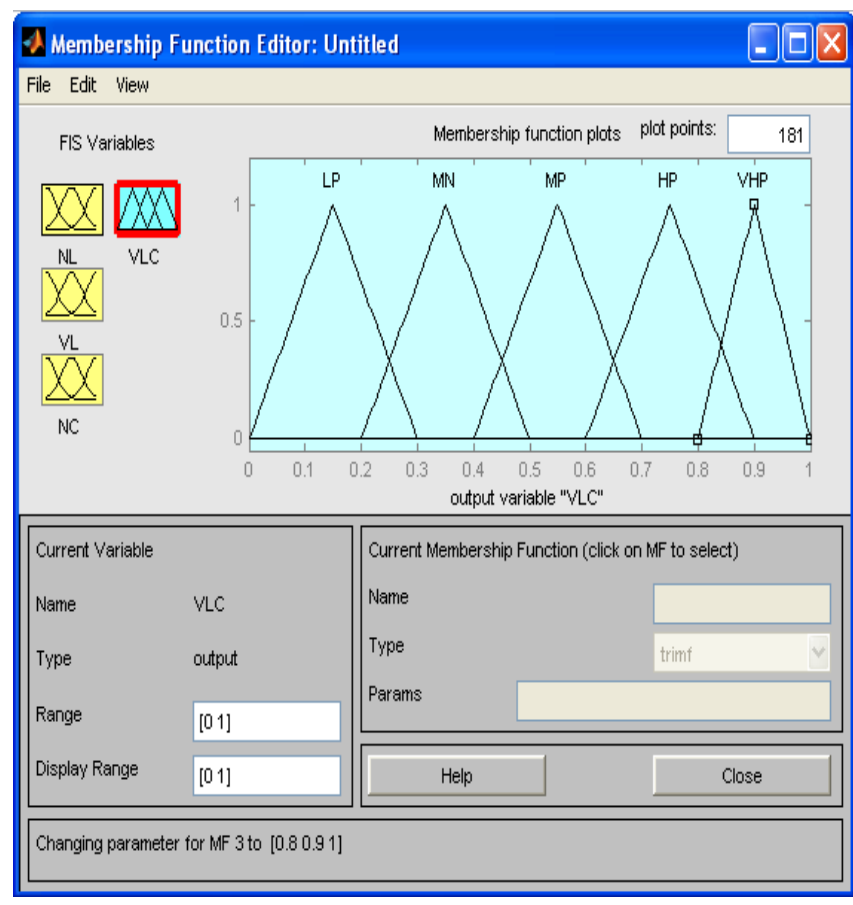

Fig4. Volume level change (VLC): VLC is defined by the triangular Fuzzy Membership functions to train the IVC.

The following forty if then fuzzy rules were defined for membership functions in fuzzy toolbox of MATLAB to design fuzzy volume controller for mobile phones. Some of them are given here as follows:

If NL is low, VL is LP and NC is Office, then VLC is LP. If $\mathrm{NL}$ is low, VL is MP and NC is Market, then VLC is LP. If NL is high, VL is HP and NC is car, then VLC is HP .

\section{RESULT AND DISCUSSION}

The output of neural background noise classifier as Noise Class (NC), and Neural noise level detector as Noise Level (NL) and Volume level (VL) from mobile phones was fed to fuzzy volume controller and corresponding volume level change (VLC) was found to vary from $5 \mathrm{db}$ to $60 \mathrm{db}$.

Range for volume level(VL) in case of human talk varies from $28 \mathrm{db}$ to $58 \mathrm{db}$ which was mixed with the four categories of internet noises from $0.1 \mathrm{db}$ to $95 \mathrm{db}$ and it was also normalized between 0 to 1 for defining the membership function using fuzzy logic toolbox.

In the proposed system, the maximum VLC (Volume level Change) was observed in case of train noise class and minimum in case of office noise class. The minimum and maximum VLC for office noise and train noise was between 0 to $12 \mathrm{db}$ and $42 \mathrm{db}$ to $62 \mathrm{db}$ respectively. For car and market noise, it was between 10 to $40 \mathrm{db}$. The overall corresponding VLC in smart volume controller varied from $10 \mathrm{db}$ to $55 \mathrm{db}$. Thus VLC (volume level change) was observed maximum in case of train and minimum in case of office. Fig 2-4 as shown in the previous section were obtained during simulation carried out using fuzzy logic toolbox available in MATLAB2018a. In the table1, FIS variables such as noise level (NL), Noise Class (NC), Volume Level (VL), Volume Level Change (VLC) were defined by using different linguistic variables and the corresponding range in terms of numerical values were defined for them.

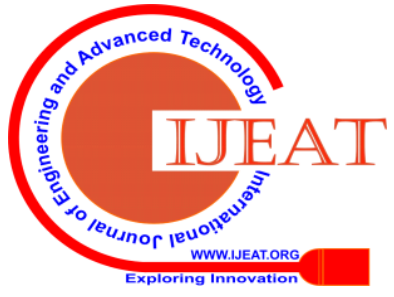


For instance, Noise level was described by linguistic variable such as low, medium and high. The low was assigned the numerical values between 0.08 to 0.4 , while medium and high were assigned the values between 0.3 to 0.7 and 0.6-1.0 respectively. Fuzzy inference system (FIS) illustrated by Fig 2 indicate the triangular membership functions named as low positive-LP (0-0.3), medium negative-MN (0.2-0.5), medium positive-MP (0.4-0.7), high positive-HP (0.6-0.9), and very high positive-VHP (0.6-1). The change in volume level was determined using the assigned variables as discussed above.

\section{CONCLUSION}

Using the proposed fuzzy based smart volume controller, maximum VLC (volume level Change) was observed in case of train noise class and minimum in case of office noise class. The minimum and maximum VLC for office noise and train noise was between 0 to $12 \mathrm{db}$ and $42 \mathrm{db}$ to $62 \mathrm{db}$ respectively. For car and market noise, VLC was observed between 10 to $40 \mathrm{db}$. The overall corresponding VLC in smart volume controller varied from $10 \mathrm{db}$ to $55 \mathrm{db}$. Thus, volume level change was observed maximum in case of train and minimum in case of office.

\section{REFERENCES}

1. Suthikshn Kumar, A review of smart volume controllers for consumer electronics, IEEE Transactions on Consumer Electronics, vol. 51, no.02, pg. 600-605, 2005.

2. B.C.Kamble, Speech recognition using Artificial Neural Network-A Review, Int. Journal of Computing, Communications \& Instrumentation Engg, vol.3, no.1, pg.1-4, 2016.

3. 3.S.G.Koolagudi, D.Rastoi, K.S.Rao, Identification of Languages using Mel-Frequency Cepstral Coefficients, Procedia Engineering, vol.38, pg.3391-3398, 2012.

4. B.Aarti, S.K.Koparapu, Spoken Indian language classification using ANN-An experimental study, 4th Int. Conference on Signal Proc. \& Integrated Network, 2-3 Feb. 2017.

5. S. Paulikas, R. Karpavicius, Applications of LPCs Interpolation in Speech Signal Coding, Elektronika ir Elektrotechnika, 80(8):39-42, 2007.

6. C.H.Huang, C.Wen, K.C.Cheng, C.L. Hsiao, Design of Smart Volume Controller for Televisions, IEEE Transactions on Consumer Electronics, vol.59, no.3, 2013.

7. O.A. Jarrah, A.Shaout, Automatic Volume Controller using Fuzzy Logic, Journal of Intelligent and Fuzzy Systems, vol. 18, no.04, pg. 329-343, 2007.

8. B. Mondal, Performance Comarison of Conventional PID and FLC in the Field of over headed water level control system, International Journal Computer Sciences and Engineering, vol. 4, no.6, pg.76-81, 2016 